\title{
BELL'S PALSY AMONG INFANTS - OUR EXPERIENCES IN A TERTIARY CARE HOSPITAL OF EASTERN INDIA
}

\author{
SANTOSH KUMAR SWAIN ${ }^{1}$, ISHWAR CHANDRA BEHERA ${ }^{2 *}$, MAHESH CHANDRA SAHU \\ ${ }^{1}$ Department of Otorhinolaryngology, IMS and SUM Hospital, Siksha "0" Anusandhan University, K8, Kalinganagar, Bhubaneswar - 751 003, \\ Odisha, India. ${ }^{2}$ Department of Community Medicine, IMS and SUM Hospital, Siksha "O" Anusandhan University, K8, Kalinganagar, \\ Bhubaneswar - 751 003, Odisha, India. ${ }^{3}$ Directorate of Medical Research, IMS and SUM Hospital, Siksha “0” Anusandhan University, K8, \\ Kalinganagar, Bhubaneswar - 751 003, Odisha, India. Email: ishwarbehera99@gmail.com
}

Received: 25 April 2017, Revised and Accepted: 19 May 2017

\section{ABSTRACT}

Objective: To know and standardize the clinical diagnosis and management of Bell's palsy among infants from a tertiary care hospital of Eastern India.

Methods: In this prospective study, we have documented detail clinical manifestation and management of infants of Bell's palsy from the pediatric age group. When an infant presents with facial nerve paralysis, a full clinical history and detailed examination were recommended for accurate diagnosis. Simultaneously, the parents were reassured, and the prognosis was explained to the parents. Oral steroids along with physiotherapy were started in the outdoor basis.

Results: We diagnosed six infants of Bell's palsy in the age group of 5 months to 12 months. Among them, 4 females and rest 2 were males. These infants showed improvement with complete recovery in $83 \%$ of cases within 3 weeks, whereas partial recovery was seen in rest.

Conclusion: In this study, we find out that oral steroids along with physiotherapy and eye care are effective for Bell's palsy in infants.

Keywords: Bell’s palsy, Infant, Facial nerve, Oral steroid.

(c) 2017 The Authors. Published by Innovare Academic Sciences Pvt Ltd. This is an open access article under the CC BY license (http://creativecommons. org/licenses/by/4. 0/) DOI: http://dx.doi.org/10.22159/ajpcr.2017.v10i9.19388

\section{INTRODUCTION}

Bell's palsy in an infant is an extremely rare clinical entity. Bell's palsy is an acute unilateral idiopathic facial nerve paralysis. Although Bell's palsy can occur at any age group, individuals between 15 and 45 years are most commonly susceptible [1]. Facial nerve paralysis whether in child or adult, it results in weakness of facial musculature, impacting verbal communication, social interaction with respect to facial expression, oral competence, taste, and protection to the cornea and vision. Facial nerve palsy can become devastating situation when it occurs in a child where parents present with an unreasonable concern for the recovery of their child. The cause of facial nerve paralysis among children is classified into infectious causes, non-infectious causes, and idiopathic. Infectious causes include otitis media, herpes zoster oticus, lyme disease, mumps, and infectious mononucleosis. Non-infectious causes are congenital, trauma, autoimmune diseases, and metabolic disorders. Idiopathic cause is the most commonly attributed to the Bell's palsy [2]. In children, the chances of Bell's palsy appear to be more frequent in cold seasons [3]. Although facial nerve paralysis is not a life-threatening situation, it can have been affect on the quality of life and cause significant functional and psychological impact on the child as well as parents. Correct diagnosis needs a complete history taking, physical examination, and imaging. Treatment requires medical therapy, surgical intervention, or combination. We herein report six cases of Bell's palsy among infants with their management.

\section{METHODS}

A total of 6 patients of Bell's palsy under the age of one year were examined at the outpatient Department of Otorhinolaryngology between January 2013 and December 2015. Out of six patients, four were female infants and two were male. Infants with congenital facial palsy or diagnosed to have middle ear infections or history of trauma to the head were excluded from this study. A detail clinical examination was done for sinus, rashes, or mass over the pinna or external auditory canal, and also any congenital abnormality was looked for. Along with facial nerve, other cranial nerves were examined. The recovery of facial nerve paralysis was assessed as per the House-Brackmann scale.

\section{Case 1}

A 9-month-old female brought to the outpatient Department of Otorhinolaryngology by her parents with a history of sudden left facial asymmetry of 1-day duration. Mother denied any history of trauma or fall. She had dripping of the milk on the left side of the mouth during feeding. She had no other ears or nasal symptoms. Otoscopy examination showed normal external auditory canal and tympanic membrane. On further examination, the child had left facial palsy with the absence of nasolabial fold (Fig. 1). She was unable to close the left eye during sleep. Other cranial examinations were within normal limit. Her complete blood counts were normal. Otoacoustic emission (OAE), tympanometry, and stapedial reflex were all normal. The child was admitted for observation and showed no worsening of facial nerve function.

Case 2

A 6-month-old female infant comes to Otolaryngology outpatient department for left side facial weakness for 1-day duration. She had no history of birth trauma or any congenital anomalies. On examination, she had left facial palsy, loss of nasolabial fold, and unable to close the left eye (Fig. 2). She had no other ear, nose, and throat symptoms. Other cranial nerves were normal. OAE and BERA were within normal limit. She was started oral steroids for 3 weeks. Her facial nerve functions recovered fully after 3 weeks.

\section{Case 3}

An 11-month-old male brought to the emergency department by his parents with complaints of right facial asymmetry of 1-day duration. 
The child had one episode of fever in the previous day and on the day of presentation was afebrile. Parents denied any history of fall or trauma. The child was active except for the dripping of the milk on the affected side of the mouth. On detail examination, the child was showing loss of nasolabial fold in the right side and unable to close the right eye (Fig. 3). Ears, nose, and throat were normal. Audiological tests were within normal limit.

\section{Case 4}

An 8-month-old male infant comes with parents to the emergency department for complaints of sudden onset of left side facial weakness. She had no history of fever or any kind of injuries. The child was showing dripping of milk during breastfeeding. There was a loss of nasolabial fold in the left side, but the eye closure was normal. Examination of ear, nose, and throat was showing no abnormality. Other cranial nerve examinations were within normal limit.

\section{Case 5}

A 12-month-old female infant comes to the outpatient department of otolaryngology with presentation of left side facial weakness. She had a history of exposure to cold. She had no fever or any history trauma. On examination, she was showing a loss of nasolabial fold in the left side, deviation of angle of the mouth to the right side during smiling, and she was unable to close the left eye (Fig. 3). There was leaking of liquid from the mouth during feeding. Examination of nose and ear was normal, and other cranial nerves were within normal limit.

\section{Case 6}

A 5-month-old female infant with her parents attended to outpatient Department of Otorhinolaryngology for the complaints of left side facial weakness. The facial weakness was sudden onset with no history of trauma. She had a history of vaccination against influenza virus 5 days back. Examination of ear, nose, and cranial nerves except facial nerve was within normal limit. Her audiological profiles were normal.

\section{Treatment}

All of the six cases started on oral steroid therapy for 3-week duration in tapering doses. Physical therapy such as massaging and exercising the infant's facial muscles is done regularly by a physiotherapist. Artificial tear was applied in the eye for keeping the eye moist, and eye pad was used for preventing any untoward injury to the cornea. Facial nerve function recovered after 3 weeks without any further intervention in all cases except case- 4 .

\section{RESULTS}

Out of six cases, five cases were completely recovered by 3 weeks with the help of oral steroids. Case 4 recovered by 6 weeks. Detailed clinical profile and outcomes after treatment were given in Table 1. Case 4 showed incomplete recovery of the facial palsy.

\section{DISCUSSION}

Bell's palsy is an acute facial paralysis affecting the seventh cranial nerve without any detectable cause. Acute facial nerve paralysis in an infant is an uncommon clinical entity, and it is considered as a pediatric emergency. The incidence of facial nerve paralysis in children varies from 5 to 21:100,000/year [1]. The mean age of pediatric group lies between 5 and 11 years with no significance between male and female [4]. Among patients with facial paralysis, Bell's palsy is the most common cause (42\%), followed by trauma (21\%), infection (13\%), congenital $(8 \%)$, and neoplasm (2\%) [5]. The most common etiology of unilateral facial nerve paralysis is Bell's palsy, also known as idiopathic facial nerve palsy. The lowest incidence of Bell's palsy is seen among patients younger than 20 years of age which is the highest incidence among 60 years of age or older population. A child presenting with sudden onset of facial weakness needed detailed history and physical examination to rule out recognizable and treatable causes. The history should be emphasized on the onset, rapidity of progression, any preceding history of viral infection or trauma, and associated systemic clinical manifestations to rule out infectious, neoplastic, or hematological or traumatic causes. Bell's palsy is usually a diagnosis of exclusion. The pathophysiology of Bell's palsy is unknown, but many reported that autoimmune system is involved for causing damage to myelin after viral infections. Herpes

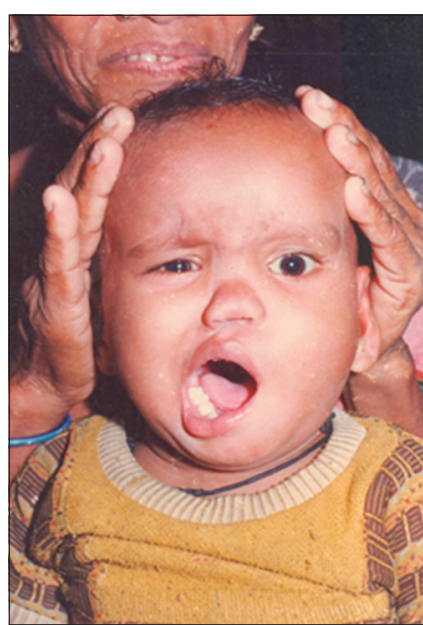

Fig. 1: A 9-month-old infant showing left side Bell's palsy

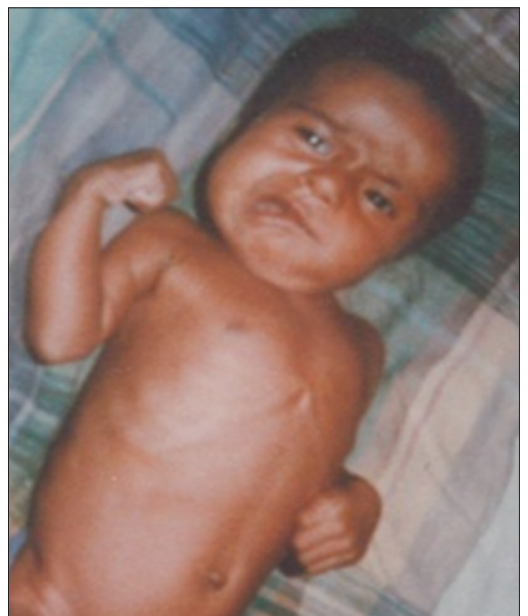

Fig. 2: A 6-month-old infant showing left side facial palsy with deviation of angle of the mouth to the right side

Table 1: Detail clinical profile and outcome of infants with Bell's palsy

\begin{tabular}{llllll}
\hline Patients & Age in months & Gender & Side of involvement & House-Brackmann grading & Duration of recovery (weeks) \\
\hline Case 1 & 9 & F & Left & Grade 3 & 3 \\
Case 2 & 6 & F & Left & Grade 2 & 3 \\
Case 3 & 11 & M & Right & Grade 2 & 3 \\
Case 4 & 8 & M & Left & Grade 4 & 6 \\
Case 5 & 12 & F & Left & Grade 3 & 3 \\
Case 6 & 5 & F & Left & Grade 3 & 3 \\
\hline
\end{tabular}




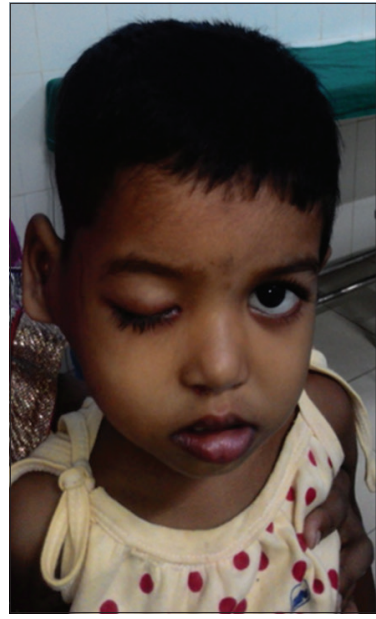

Fig. 3: A 12-month infant showing the left side Bell's palsy

simplex virus (HSV) is implicated as the cause of inflammation of facial nerve in Bell's palsy [6]. For the children with Bell's palsy and their families, the sudden impairment of facial nerve is often shocking. Hence, always high expectations by parents are placed in front of physicians for recovery. In Bell's palsy, clinical manifestations are seen as flattening of the forehead and nasolabial fold on the affected side of facial palsy, eyebrow sagging, unable to close the eye, and drawing of angle of the mouth to the non-affected side. Facial nerve paralysis can cause failure of eye closure, which without timely intervention can lead to corneal ulceration, scarring, and vision loss [7]. Uncomplicated Bell's palsy may not require further investigation in children. However, further testing is advised when infant presents with complete paralysis or showing no signs of recovery within 3 weeks of onset [5]. Topodiagnostic tests such as Schirmer's tear test and stapedial reflex test and evaluation of salivation are performed to find the location of nerve injury. Intact stapedial reflex in cases of incomplete facial nerve paralysis or return of stapedial reflex within 3 weeks of onset predicts the complete recovery of the facial nerve by 3-8 weeks [8]. Although imaging such as magnetic resonance imaging is not routinely indicated, it is advised in patients of Bell's palsy whose facial nerve paralysis is persisting more than 3 months, recurrent facial palsy, or single facial nerve segment involvement to rule out any facial nerve tumor like neuroma or any underlying neurologic diseases [3]. The electrophysiological tests such as electroneuronography, electromyography, and blink reflex are useful to assess the prognostic significance as these provide a more accurate and objective prediction of subsequent improvement. These electrophysiological tests are rarely reported in children because majority of the children recovered and seldom referred to neurophysiologist [9].

Often the concerns for the parents of pediatric Bell's palsy are whether its complete recovery can be expected. Hence, the prognosis is to be explained and reassurance will be given, which are the important part of the management. It has been thought that the inflammation and edema of the facial nerve in Bell's palsy most likely initiated by infection with HSV [9]. Hence, the common treatment approach in Bell's palsy includes steroids to reduce the inflammations of the facial nerve and acyclovir for treating the viral infection. Surgery sometimes done to prevent compression of the facial nerve, and finally eye care is also an important aspect of treatment in Bell's palsy. The treatment of idiopathic facial nerve paralysis is often controversial. The use of corticosteroids at early onset significantly improves the chance of complete recovery [10]. As in majority of cases, recovery occurs with or without the use of steroids. Acyclovir with or without steroids has also been used for the treatment of Bell's palsy because reactivation of HSV has been implicated as the cause. The prognosis of Bell's palsy is generally excellent. Most children will recover to normal within 2-4 weeks [11]. Steroids are usually safe drug for treatment of Bell's palsy. We avoided acyclovir in our cases and only oral prednisolone given. In these cases, we started syrup prednisolone $1 \mathrm{mg} / \mathrm{kg}$ /day with tapering dose for 21 days. Early treatment with oral steroids is a very effective step for improvement of facial musculature functions. For eye care, artificial tears, ointment, humidifying cover, eyelid implant, botulinum toxin, or eyelid stitches can be useful. The mean duration of recovery in this study was 3.5 weeks which is comparable to that Dhiravibulya (6.61 weeks) [12] and Sunil Kumar (6.5 weeks) [13] where they treated with multivitamins as placebo along with physiotherapy without oral steroids. Mastoid surgery has no role in these cases $[14,15]$.

\section{CONCLUSION}

Although Bell's palsy is the most common peripheral facial nerve paralysis, it is a rare occurrence among pediatric population and more rare particularly in infants. Even though it is not a life-threatening condition, it can have severe effects on patient's quality of life. The diagnosis of Bell's palsy in an infant should be a diagnosis of exclusion. Diagnosis is primarily based on clinical presentation which includes incomplete eye closure, weak eyebrow lifting, drooping mouth corner, dry eye, loss of taste sensation, hyperacusis, and pain in the ear. Even it is a self-limiting clinical condition with a favorable prognosis; we should not delay the treatment. Early treatment with a course of oral corticosteroid for 2-3 weeks after onset of Bell's palsy usually provides benefit to the patients.

\section{Study limitation}

This study has a relatively small sample size and may limit the outcome of the above interpretation. However, the outcome of this study will encourage the use of oral steroids along with facial physiotherapy in case Bell's palsy among infants. Additional eye care with artificial tear will prevent exposure keratitis.

\section{REFERENCES}

1. Jenke AC, Stoek LM, Zilbauer M, Wirth S, Borusiak P. Facial palsy: Etiology, outcome and management in children. Eur J Paediatr Neurol 2011;15(3):209-13.

2. Shih WH, Tsen FY, Yeh TH, Hsu CJ, Chen YS. Outcomes of facial palsy in children. Acta Otolaryngol 2009;129:915-20.

3. Tsai HS, Chang LY, Lu CY, Lee PI, Chen JM, Lee CY, et al. Epidemiology and treatment of Bell's palsy in children in Northern Taiwan. J Microbiol Immunol Infect 2009;42(4):351-6.

4. Chen WX, Wong V. Prognosis of Bell's palsy in children and analysis of 29 cases. Brain Dev 2005;27(7):504-8.

5. Singh P, Jain V. Bell's palsy in children. Semin Pediatr Neurol 2003;10(4):289-97.

6. Furuta Y, Fukuda S, Chida E, Takasu T, Ohtani F, Inuyama Y, et al. Reactivation of herpes simplex virus Type 1 in patients with Bell's palsy. J Med Virol 1998;54:162-6.

7. Lane C. Management of ocular surface exposure. Br J Ophthalmol 2012;96(4):471-2.

8. Finsterer J. Management of peripheral facial nerve palsy. Eur Arch Ototlaryngol 2008;265(7):743-52.

9. Wong V. Outcome of facial nerve palsy in 24 children. Brain Dev 1995;17:294-6.

10. Worster A, Keim SM, Sahsi R, Pancioli AM. Best evidence in emergency medicine (BEEM) group. Do either corticosteroids or antiviral agents reduce the risk of long-term facial paresis in patients with new-onset Bell's palsy? J Emerg Med 2010;38:518-23.

11. Evans AK, Licameli G, Brietzk S. Pediatric facial nerve paralysis: Patients management and outcomes. Int J Pediatr Otorhinolaryngol 2005;69:1521-8.

12. Dhiravibulya K. Outcome of Bell's palsy in children. J Med Assoc Thai 2002;85:334-9.

13. Kumar S, Garg S, Mittal A, Sahni JK. Bell's palsy in early childhood: A series of six cases. Indian J Otol 2012;18:140-2.

14. Nalini R, Ezhilramya J. A comparative study of efficacy and safety of lornoxicam and diclofenac as postoperative analgesics after mastoidectomy surgery. Int J Pharm Pharm Sci 2017;9(2):77-83.

15. Mathews L, Roy A. Management of pain using transdermal patches-a review. Asian J Pharm Clin Res 2016;9:32-5. 